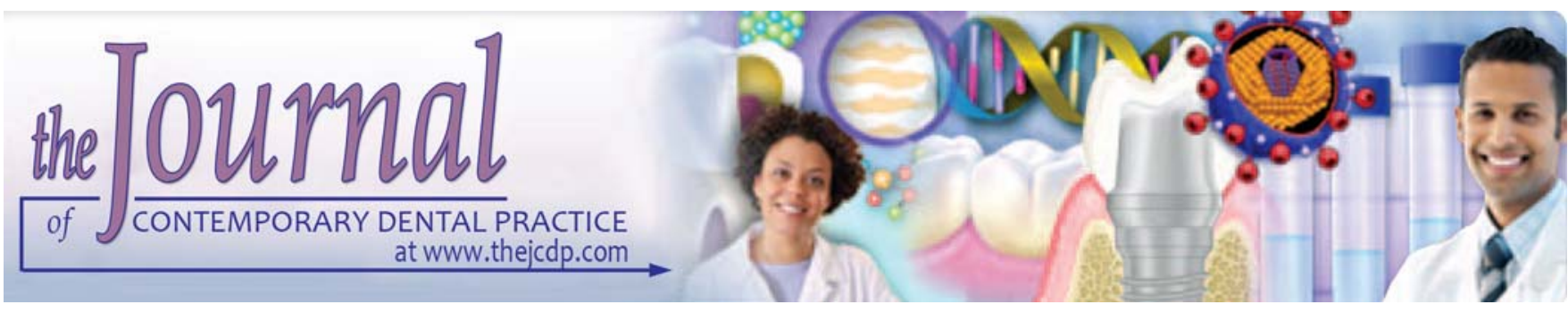

\title{
Lateral Radiographic Technique: An Alternative Approach for Anterior Teeth
}

Carlos Vieira Andrade Junior, Emmanuel João Nogueira Leal Silva, Juliana Melo Da Silva, Caio Cesar Randi Ferraz

\section{ABSTRACT}

Aim: To describe the lateral radiographic technique, its use and indication.

Background: The clinician's difficulty in performing the correct diagnosis for some endodontic situations sometimes leads to a compromised treatment of some teeth. Considering this, an alternative radiographic technique for anterior teeth (lateral radiographic technique - LRT) may be used to help endodontic diagnosis.

Technique: This technique use a periapical radiographic film or sensor that is placed vertically in the anterior portion of maxilla or mandible and with the beam passing perpendicular to the radiographic film its possible to evaluate the possibility of a fenestration on the buccal cortical bone plate; differentiate overextension and overfilling; indentify root fractures; confirm the presence and location of an instrument in the root canal system; and assess the treatment of root perforations.

Conclusion: LRT is an easy and cheap technique that can be used to help in anterior teeth diagnosis.

Clinical significance: Considering that LRT is easy to perform and available at low cost, it could be utilized more by clinicians in the dental office in order to quickly assist in diagnosis. As a complimentary radiographic examination for anterior teeth, it could become a useful supplement to aid the clinical practice.

Keywords: Endodontics, Radiology, Diagnostic radiology.

How to cite this article: Andrade CV J r, Leal Silva EJ N, Da Silva J M, Ferraz CCR. Lateral Radiographic Technique: An Alternative Approach for Anterior Teeth. J Contemp Dent P ract 2013;14(1):43-46.

\section{Source of support: Nil}

Conflict of interest: None declared

\section{BACKGROUND}

The relationship between the endodontic and radiology specialties is close since the use of images obtained by radiographs is necessary for all stages of the endodontic treatment. The greatest difficulty that clinicians face when evaluating the radiographic images is the superimposition of the anatomical structures due to its two-dimensional characteristic, which frequently make the diagnosis difficult. ${ }^{1}$ In spite of this limitation, intraoral radiographic examinations are commonly used by dentists and they are divided into three main categories: Periapical, bitewing and occlusal radiographs. ${ }^{2}$

In some clinical situations such as root fracture, presence of bone fenestration, dental perforations and anatomical anomalies, it may be difficult to reach a diagnosis using conventional periapical radiography. For these situations, techniques that allow a three-dimensional (3D) visualization without superimposition of the anatomical structures are better. ${ }^{3} \mathrm{~A}$ good example of a 3D image exam is the conebeam computed tomography ( $C B C T$ ) that allows the visualization in the axial and proximal planes. However, CBCT is expensive and the technology is usually available only in large metropolitan centers, which makes its implementation more limited. ${ }^{4}$

For anterior teeth, the lateral radiographic technique may be a feasible and efficient resource for endodontic diagnosis, particularly as it is easy to perform and inexpensiveto acquire. Thus the objective of this article is to describe the lateral radiographic technique, its use and indications.

\section{TECHNIQUE}

The lateral radiographic technique (LRT) may be used in both maxillary and mandibular anterior teeth.

\section{Maxilla}

1. The patient must be placed where the median sagittal plane is perpendicular and the occlusal plane (maxilla) is parallel to the ground (Fig. 1A).

2. The radiographic film or sensor should be placed vertically between the upper lip and the anterior portion of the maxilla (Fig. 1B). The patient is instructed to hold the film with the finger. 


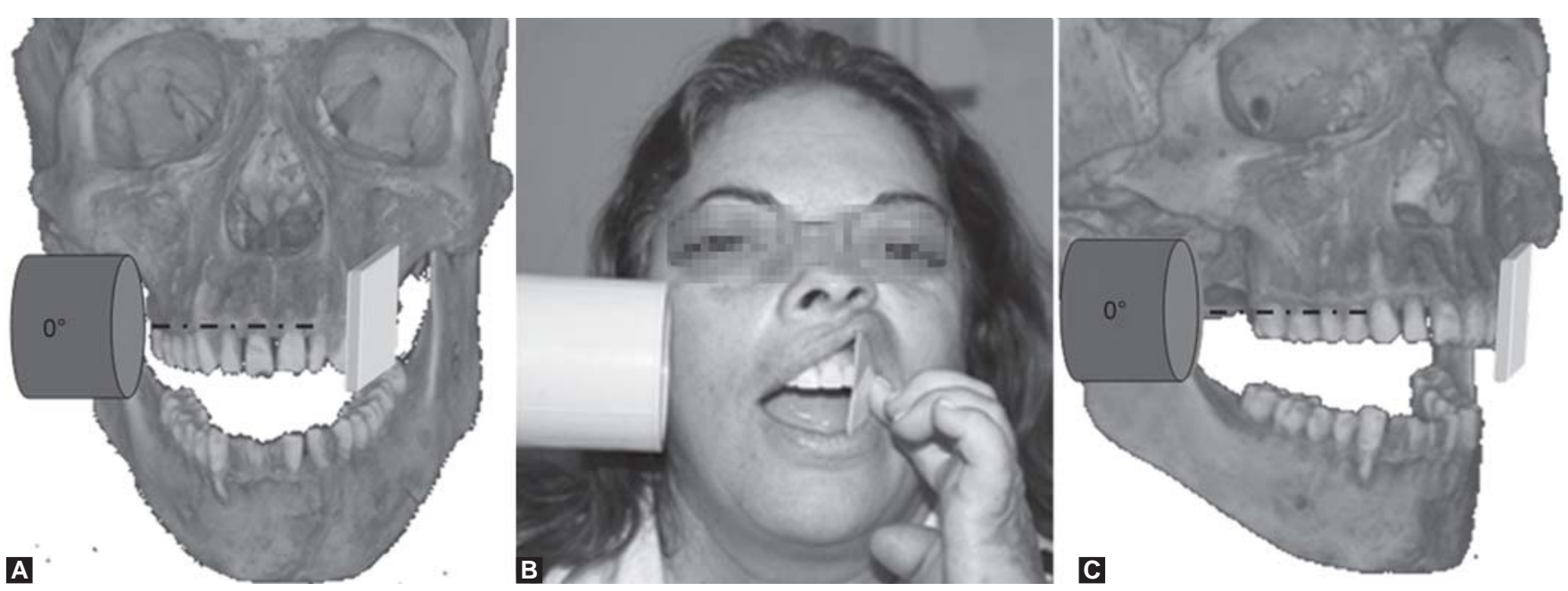

Figs 1A to C: (A) LRT for the maxilla; $(B)$ clinical application of LRT; $(C)$ variation of the technique for lateral teeth and canines

3. The X-ray beam should be placed on the opposite side of the film or sensor with a horizontal angulation of $0^{\circ}$. Vertical angulation must be placed so that the beam passes perpendicular to the radiographic film (Fig. 1B).

4. The exposure time is the same used for conventional periapical radiographs, vary depending on the type of radiographic film used. If using digital radiographs, an adjustment in exposure time may not be needed since the image can be adjusted by manipulation of the digitalized image.

5. The patient should be instructed to rotate their head so the teeth are in the proper position. The beam should be tangential to the point on the line of the dental arch corresponding to the specific tooth of interest. This would be essential to limit superimpositions (Fig. 1C).

\section{Mandible}

The technique is the same as described with only one exception: The mandibular occlusal plane should be parallel to the ground. To do this, draw a line from the tragus to the labial commissure and orient the seated patient so this is parallel to the ground (Fig. 2).

Some possible indications for this technique could be:

1. To evaluate the possibility of a fenestration on the buccal cortical bone plate;

2. To differentiate overextension and overfilling;

3. To potentially indentify root fractures;

4. To confirm the presence and location of an instrument in the root canal system;

5. To assess the treatment of root perforations.

To better illustrate indications for this technique, three cases will be presented where LRT was used to confirm the final diagnosis.

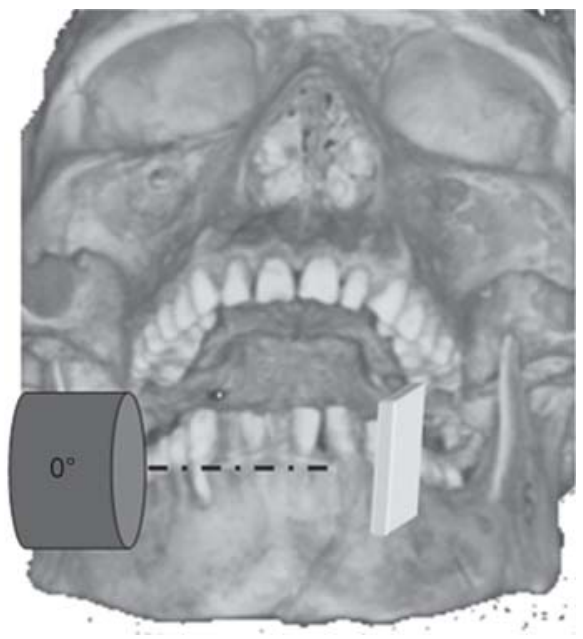

Fig. 2: LRT for the mandible

\section{Case 1}

Patient M F presented to the dental office reporting previous endodontic retreatment of teeth 11 and 21 , but still felt sensitivity to palpation. Radiographic examination showed that the length of the teeth were below average (Fig. 3A). U pon pal pation the patient related a feeling that the root of the tooth was being touched. The suspicion that the bone was fenestrated was confirmed by LRT (Fig. 3B).

\section{Case 2}

The patient MRO had an inflammatory cystic lesion involving the apices of teeth 12, 11, 21 and 22 which occurred before endodontic treatment was perfomed. A fter treatment was completed, it was suspected that the obturation was overextended (Fig. 4A). U sing LRT, it was found that there were only extravasations of endodontic sealer (Fig. 4B). 


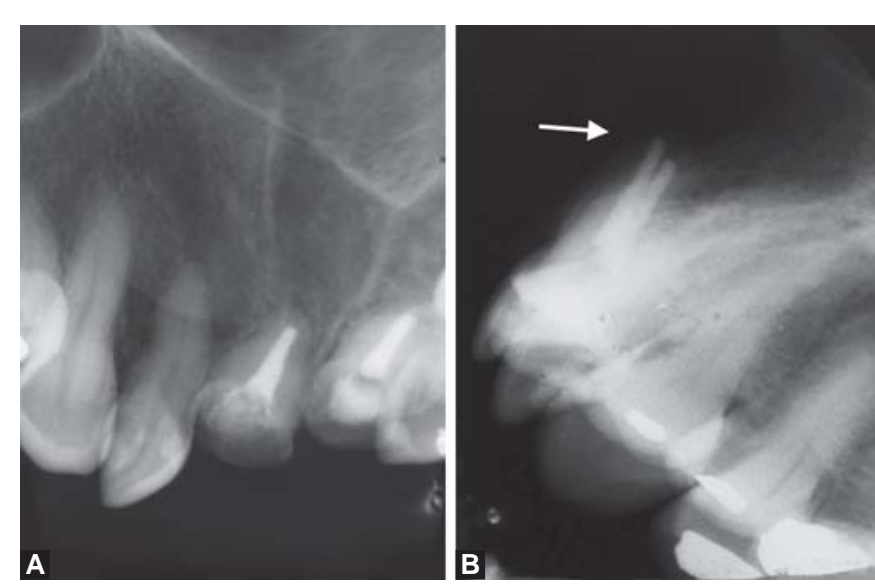

Figs 3A and B: (A) Initial radiograph;

(B) LRT showing fenestration
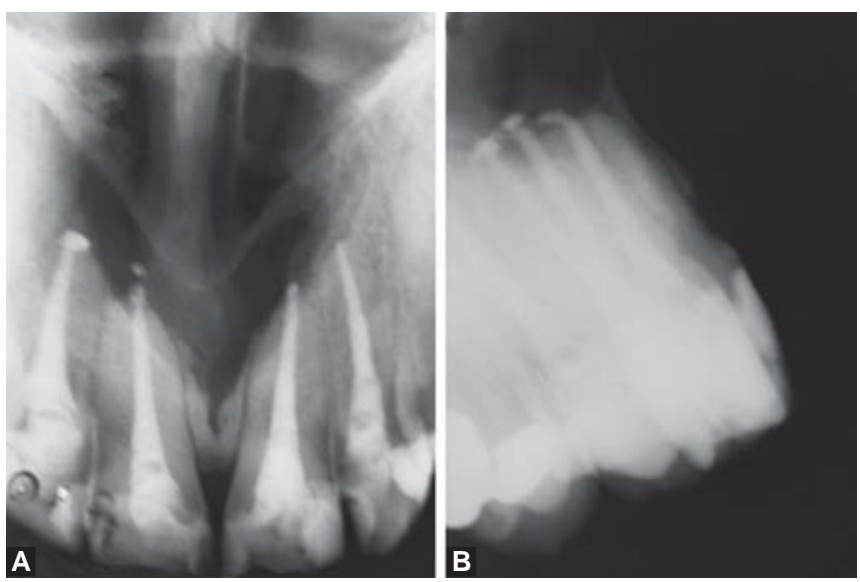

Figs 4A and B: (A) Final radiograph showing extravasations of filling material; (B) LRT showing extravasations of root canal sealer

\section{Case 3}

The radiograph examination from the patient $V R F R$ showed an image suggesting internal resorption in tooth 21 (Fig. 5B). With the aid of LRT, it was possible to verify that the external surface of the root was not perforated by the
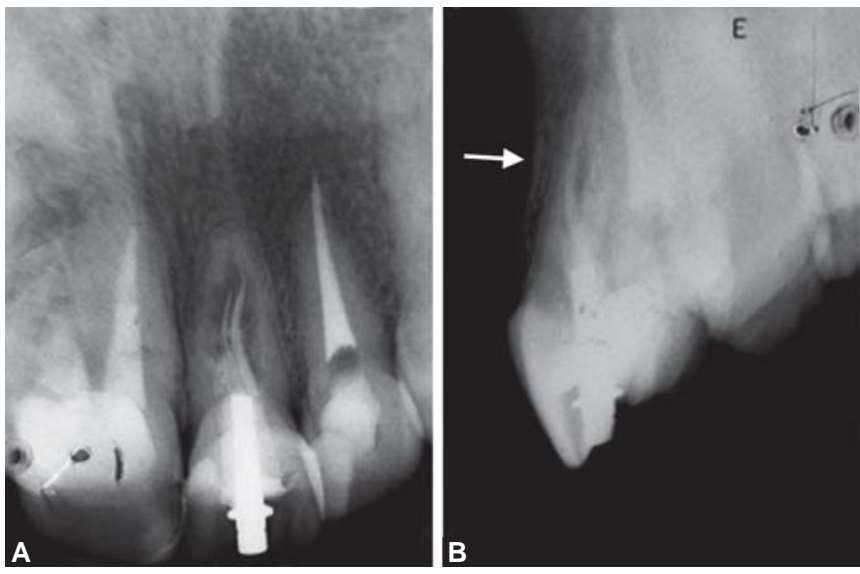

Figs 5A and B: (A) Initial radiograph showing an image suggesting dental resorption; (B) LRT showing integrity of vestibular portion of the root resorption (Fig. 5B) and thus no communication with the periodontal space.

\section{DISCUSSION}

Considering that LRT is easy to perform and available at low cost, it could be utilized more by clinicians in the dental office in order to quickly assist in diagnosis. This would also decrease the need to refer the patient for CBCT examination. There is little doubt that the benefits offered by CBCT to the endodontic practice, ${ }^{4}$ but the high cost of this examination must be taken into consideration especially, if there is a less expensive alternative offering comparable diagnostic quality.

On performing a search of the dental literature for $L R T$, it was found that $\mathrm{O}^{\prime} \mathrm{C}$ onnor et al presented a clinical case showing the application of a very similar technique to LRT to help determine the position of an endodontic instrument during the treatment of calcified root canals. ${ }^{5}$ How ever, the authors do not describe the steps needed to perform the technique nor other possible indications common to clinical practice.

If dentists begin to routinely utilize LRT, new applications could be developed to help an endodontic diagnosis. It is important to note that the authors do not advocate that LRT replace the conventional periapical radiographs or supplant situations where it would be insufficient and only CBCT able to provide adequate image details.

\section{CONCLUSION}

As a complimentary radiographic examination for anterior teeth LRT could become a useful supplement to aid the clinical practice.

\section{CLINICAL SIGNIFICANCE}

Considering that LRT is easy to perform and available at low cost, it could be utilized more by clinicians in the dental office in order to quickly assist in diagnosis. As a complimentary radiographic examination for anterior teeth, it could become a useful supplement to aid the clinical practice.

\section{REFERENCES}

1. Goldman M, Pearson A, D arzenta N . R eliability of radiographic interpretations. Oral Surg 1974;38:340.

2. Tetradis S, A nstey P, Graff-Radford S. Cone-beam computed tomography in the diagnosis of dental disease. Tex Dent J 2011;128:620-28.

3. L ofthag-Hansen S, Huumonen S, Grondahl K, Grondahl HG. Limited cone-beam CT and intraoral radiography for the diagnosis of periapical pathology. Oral Surg Oral Med Oral Pathol Oral Radiol Endod 2007;103:114-19. 
4. Cotton TP, Geisler TM, Holden DT, Schwartz SA, Schindler WG. Endodontic applications of cone-beam volumetric tomography. J Endod 2007;33:1121-32.

5. O'Connor RP, D eM ayo TJ , R oahen J O. The lateral radiograph: An aid to labiolingual position during treatment of calcified anterior teeth. J Endod 1994;20:183-84.

\section{ABOUT THE AUTHORS}

\section{Carlos Vieira Andrade Junior}

Professor, Department of Endodontics, UESB, B razil

\section{Emmanuel João Nogueira Leal Silva}

(Corresponding Author)

Professor, Endodontics Specialty UERJ, Rio de Janeiro State University, B razil, e-mail: nogueiraemmanuel@ hotmail.com

\section{Juliana Melo Da Silva}

Professor, Department of Endodontics, UFPA, PA, B razil

\section{Caio Cesar Randi Ferraz}

Professor, Department of Endodontics, UNICAM P, SP, B razil 